\title{
BMJ Open The spectrum of unhealthy drug use and quality of care for hypertension and diabetes: a longitudinal cohort study
}

\author{
Theresa W Kim, ${ }^{1}$ Jeffrey H Samet, ${ }^{1,2}$ Debbie M Cheng, ${ }^{1,3}$ Judith Bernstein, ${ }^{2,3}$ \\ Na Wang, ${ }^{4}$ Jacqueline German, ${ }^{1}$ Richard Saitz ${ }^{1,2}$
}

\begin{abstract}
To cite: Kim TW, Samet JH, Cheng DM, et al. The spectrum of unhealthy drug use and quality of care for hypertension and diabetes: a longitudinal cohort study. BMJ Open 2015;5:e008508. doi:10.1136/bmjopen-2015008508
\end{abstract}

- Prepublication history for this paper is available online. To view these files please visit the journal online (http://dx.doi.org/10.1136/ bmjopen-2015-008508).

Received 22 April 2015 Revised 8 October 2015 Accepted 16 October 2015

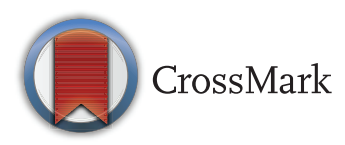

${ }^{1}$ Clinical Addiction Research and Education (CARE) Unit, Section of General Internal Medicine, Boston Medical Center, Boston University School of Medicine, Boston, Massachusetts, USA

${ }^{2}$ Department of Community Health Sciences, Boston

University School of Public Health, Boston,

Massachusetts, USA

${ }^{3}$ Department of Biostatistics, Boston University School of Public Health, Boston, Massachusetts, USA

${ }^{4}$ Data Coordinating Center, Boston University School of Public Health, Boston,

Massachusetts, USA

Correspondence to Dr Theresa W Kim; theresa.kim@bmc.org

\section{ABSTRACT}

Objectives: Although it is well known that addiction is associated with adverse medical consequences, the effects of the spectrum of unhealthy drug use (illicit drug or prescription misuse) on chronic conditions such as hypertension and diabetes are understudied. This study evaluated the associations between measures of drug use (ie, frequency, severity and type) and standard quality metrics for inadequate blood pressure (BP) and blood glucose (BG) control.

Design: Longitudinal cohort study.

Participants: Adult primary care patients with unhealthy drug use and hypertension or diabetes.

Setting: Urban hospital-based primary care practice.

Measurements: Outcomes were (1) inadequate BP (systolic BP $\geq 140$ or diastolic $B P \geq 90$ ) and (2) inadequate $B G$ (glycated haemoglobin $\geq 8 \%$ ) control (Healthcare Effectiveness Data and Information Set criteria). Drug use was characterised by a primary independent variable, drug use frequency, and two secondary variables, severity of use and drug type. We fit separate regression models for each drug use measure and outcome.

Results: Overall, 40\% (65/164) of the sample with hypertension had inadequate BP control and 44\% (24/ 54 ) of those with diabetes had inadequate BG control. More frequent drug use was not significantly associated with inadequate BP control (adjusted OR (AOR) 0.67; $95 \% \mathrm{Cl} 0.31$ to 1.46 , highest vs lowest tertile; AOR $0.72 ; 95 \% \mathrm{Cl} 0.36$ to 1.41 , middle vs lowest tertile) or BG control (AOR 0.27; 95\% Cl 0.07 to 1.10, highest vs lowest tertile; AOR 1.01; $95 \% \mathrm{Cl} 0.38$ to 2.69 , middle vs lowest tertile). Drug use severity was also not associated with BP or BG control. Cocaine use was associated with inadequate $B G$ control compared to marijuana use (AOR 8.82; 95\% Cl1.86 to 41.90).

Conclusions: Among primary care patients with recent drug use and hypertension or diabetes, drug type was significantly associated with inadequate BG, but not BP control. Frequency and severity of use were not significant predictors of either outcome.

\section{INTRODUCTION}

An estimated 24.6 million Americans or $9.4 \%$ of the US population over the aged

\section{Strengths and limitations of this study}

- This study examined primary care patients with hypertension or diabetes and unhealthy drug use and-a group that has not been well-studied despite the need for strategies to improve the care of chronic conditions in patients with multimorbidity.

- The impact of a wide spectrum of drug use was examined from infrequent use without consequences to severe use disorder.

- The outcome was disease control, a clinically significant but a more difficult performance measure to achieve than receipt of recommended care such as routine eye or podiatry care.

- Power to detect differences was limited due to the sample size.

- Marijuana was the predominant drug of choice for a large proportion of the sample so examination of these issues in other samples with more stimulant or opioid use may yield different results.

12 years have used an illicit drug in the past month according to the 2013 National Survey on Drug Use and Health. ${ }^{1}$ More than two-thirds of drug use is hazardous (nondependent) use, yet much of the literature on the health impact of drug use focuses on drug use disorders. Non-dependent drug use is difficult to examine because there are no corresponding International Classification of Diseases, Ninth Revision (ICD-9) diagnosis codes in the billing databases used by many studies. It is also limited by ability of clinical staff in detecting and documenting drug use. The few studies that have used validated screening questions to identify a broad spectrum of drug use have not found a consistent association between drug use and health outcomes that include overall health status, ED utilisation and access to preventing services. $^{23}$

Understanding the relationship of unhealthy drug use to medical outcomes is 
of particular importance given the large-scale initiatives by health insurers, accrediting organisations and others underpayment reform to assess the 'quality' of medical care. ${ }^{45}$ There has been a particular focus on common medical diseases that confer cardiovascular risk such as hypertension and diabetes with well-established performance measures designed to measure treatment effectiveness. ${ }^{6} 7$

Individuals with drug use are at greater risk for worse hypertension and diabetes outcomes for a number of reasons. Patient-related factors include limited access to care, poor follow-up and suboptimal self-management skills. ${ }^{78}$ Depression $^{9}{ }^{10}$ and unhealthy alcohol use $\mathrm{e}^{11} 12$ are associated with lower receipt of comprehensive diabetes care and glycaemic control. Clinician factors such as negative views of patients with drug use-best described for those with more severe or obvious use and disorders ${ }^{13}$ - may affect the quality of care of chronic diseases as has been demonstrated for preventive care. ${ }^{14}$ Addressing unhealthy drug use may compete for attention with demands of chronic illness management during clinic visits. Adverse outcomes may also result from direct effects of specific drugs such as cocaine and heart disease.

Assessment of the impact of drug use on chronic medical conditions requires data on characteristics of drug use beyond absence or presence. Drug use that differs in frequency, type of drug or severity may have different impacts on health outcomes, however, data containing such measures are difficult to obtain. Frequency of drug use may correlate with worse disease control because time spent on drug-related activities like looking for drugs may come at the expense of time for picking up medication refills, adequate nutrition ${ }^{15}$ or attending medical visits. It also may indicate greater exposure to the drug itself. In a study of hospitalised opioid-dependent patients, more days of heroin use was associated with lower physical functioning. ${ }^{16}$ Higher doses of prescribed opioids may result in worse glycaemic control. ${ }^{17}$ More severe substance use (ie, substance use disorder) is associated with inadequate receipt of diabetes care. ${ }^{9} 18$ These studies did not specify the type of drug despite the fact that marijuana, cocaine and opioids may individually have no effect or a wide range of effects on blood pressure (BP) and blood glucose (BG) metabolism and possibly on patient behaviours such as adherence or visit attendance. The primary objective of this study was to evaluate the association of frequency of drug use and the quality of care for hypertension and diabetes among primary care patients who screened positive for drug use. Secondary objectives were to examine other measures of drug use (drug type and severity) with respect to the same indices of quality of care for hypertension and diabetes. Our hypothesis was that higher number of days used, type of drug (heroin/cocaine use), and more severe drug use are factors associated with inadequate $\mathrm{BP}$ and $\mathrm{BG}$ control.

\section{METHODS}

\section{Study design and participants}

This was a secondary analysis of the Assessing Screening Plus brief Intervention's Resulting Efficacy to stop drug use (ASPIRE) study, a randomised trial that tested the efficacy of brief intervention counselling in primary care patients identified with unhealthy drug use (illicit drug use or prescription drug misuse). ${ }^{19}$ The study took place at an urban hospital-based primary care practice that serves a predominantly lower income population of the metro Boston area.

Adults presenting for an appointment with a primary care clinician were screened for unhealthy drug use by a health educator employed by the hospital or research assistant trained in the same role. Eligibility criteria included a drug-specific Alcohol, Smoking and Substance Involvement Screening Test (ASSIST) score $\geq 2$ indicating any drug use in the past 3 months (marijuana, cocaine, opioids or other drugs).$^{20}$ Patients were excluded from the parent study if they did not speak English or Spanish, had specific plans to leave the Boston area that would prevent research participation, could not provide contact information for tracking purposes, or were pregnant. The ASPIRE study found no efficacy for brief intervention counselling on drug use, drug use consequences and other health measures. ${ }^{19}$

The current study included two separate subsamples. The first was comprised of study participants with hypertension ascertained by diagnostic billing code or presence on electronic medical record (EMR) problem list and a BP measurement no more than 30 days before study enrolment. The second subsample included those with diabetes, ascertained by billing code or presence on the EMR problem list and a glycated haemoglobin (HbA1c) serum measurement no more than 90 days before study enrolment.

The Institutional Review Board of Boston University Medical Center approved this study. Additional privacy protection was secured by the issuance of a Certificate of Confidentiality by the Department of Health and Human Services. All participants provided written informed consent.

\section{MEASURES}

\section{Outcomes}

The two study outcomes, inadequate BP and BG control, were defined as failure to meet criteria for 'good BP control' and 'good BG control' using the specifications of the National Committee for Quality Assurance (NCQA) and Healthcare Effectiveness Data and Information Set. ${ }^{7}$

Inadequate BP control was defined as systolic BP of 140 or higher or diastolic BP of 90 or higher. BP data were analysed if obtained during a medical visit no more than 30 days before the interview at study entry (when drug use was assessed). Study participants also contributed data for analysis if $\mathrm{s} / \mathrm{he}$ had a medical visit (during 
which $\mathrm{BP}$ was measured) no more than 30 days before the 6-month study interview. Baseline and 6-month data were analysed as repeated measures data.

Inadequate BG control was defined as $\mathrm{HbAlc}$ of $8 \%$ or higher. Similar to analyses of BP control, HbA1c was collected as part of usual medical care and used in analyses of BG control if measured no more than 90 days before the interview in which drug use was assessed (ie, at study entry or the 6-month interview). If multiple BPs or HbAlc measures were available, the measurement closest to the date of the study assessment was used.

\section{Independent variables}

The primary independent variable 'days of drug use' was the number of days use in the past month of the main drug ("which substance in the past month concerns you the most?'). This was assessed with the wellvalidated 30-day Timeline Follow- back. ${ }^{20}$ In secondary analyses, two other measures of drug use were examined: drug type (marijuana, opioids, cocaine, and other, primarily benzodiazepines) and drug use severity as defined by the ASSIST global drug score. ${ }^{21}$ To avoid the assumption of linearity, days of drug use and drug use severity were categorised based on tertiles of their distributions. In the hypertension sample, the number of days of drug use in the past month was 0-2 days for the lowest tertile; 3-22 days for middle tertile and 23-30 days for the highest tertile. For the diabetes analysis, the lowest tertile was $0-4$ days, the middle tertile 5-18 days, and highest tertile or 19-30 days. Potential confounders included as covariates in analyses were demographics, health insurance (any vs none), medical comorbidity (Charlson Comorbiity Score of 1 or greater), ${ }^{22}$ and depression (Patient Health Questionnaire (PHQ-9) score of 10 or greater. ${ }^{23}$

\section{Statistical analysis}

Descriptive statistics are presented to summarise the characteristics of each analytic sample (hypertension and diabetes). To examine the association of each measure of drug use with BP control, we initially fit separate longitudinal logistic regression models for: (1) days of drug use (2) drug type and (3) drug use severity predicting inadequate BP control. Participants contributed either one or more observations to the analysis depending on the availability of $\mathrm{BP}$ or glycosylated haemoglobin (HgbAlc) data that met the inclusion criteria (ie, measurement within 30 days before the study interview for the hypertension analyses and within 90 days for the diabetes analyses). All available data at study entry and 6-month interviews were analysed as repeated measures data. Generalised estimating equations (GEE) logistic regression models were used to evaluate the association between each measure of drug use and outcome at each assessment (ie, baseline, 6 months), accounting for the correlation from using repeated observations from the same participant. An independence working correlation was used and empirical SEs from the GEE approach are reported.

For the analyses of BP control, multivariable regression models included the following covariates: age, gender, race/ethnicity, health insurance, medical comorbidity and depression. We also examined the results of one final 'fully adjusted' model with these covariates, the primary independent variable, days of drug use and other measures of drug use (ie, drug type and severity). Given the possibility that the relationship between days of drug use and BP control might differ based on other factors, exploratory analyses evaluated the following three potential effect modifiers: drug type, unhealthy alcohol use (Alcohol Use Disorders Identification Test-C) score of 3 or greater for women, 4 or greater for men) and depression. ${ }^{24}$ Separate models were fit testing the interaction between number of days drug use and each potential effect modifier. Although brief intervention counselling was not associated with less drug use (Saitz et al 2014), given the possibility that the intervention had effects on BP or BG control, we added randomisation group as a covariate in confirmatory regression models.

Similar methods were used for analyses of BG control except that multivariable regression models adjusted only for age, gender, and race/ethnicity, given the limited number of events and the smaller sample size. In secondary, exploratory analyses, we also examined days of drug use predicting both outcomes as continuous measures (ie, systolic BP and HbA1c) using separate mixed effects linear regression models. Reported $\mathrm{p}$ values were two-tailed and considered statistically significant if $<0.05$. Analyses were completed using SAS/STAT software, V.9.3.

\section{RESULTS}

\section{Study participants}

Of the 589 participants, 28\% ( $\mathrm{n}=167)$ had hypertension. Of those with hypertension, 98\% (164/167) had a BP measurement within 30 days of a study interview. Of the 589 participants, $13 \% \quad(\mathrm{n}=77)$ had diabetes. Of those with diabetes, $81 \%(62 / 77)$ had a HbAlc measurement within 90 days of a study interview comprising the diabetes study sample. There were no significant differences between participants with and without a HbAlc measurement in age, sex, race/ethnicity, main drug and days of use of the main drug (results not shown).

Baseline sociodemographic, health and drug use characteristics are displayed in tables 1 and 2 for participants in the hypertension and diabetes samples, overall and stratified by days of drug use in tertiles. Both study samples were comprised of older individuals (mean age 51 for the hypertension sample and 48 for the diabetes sample), predominantly male and non-white. About one-third did not graduate from high school. A similar proportion had significant depressive symptoms. Marijuana was the main drug for the majority $(60 \%)$ of 
Table 1 Baseline characteristics of participants with hypertension, overall and stratified by baseline number of days of drug use in the past 30 days

\begin{tabular}{|c|c|c|c|c|}
\hline \multirow[b]{2}{*}{ Characteristic } & \multirow[b]{2}{*}{ All $(n=164)$} & \multicolumn{3}{|l|}{ (tertiles) $^{x}$} \\
\hline & & Lowest $(n=44)$ & Middle $(n=63)$ & Highest $(n=57)$ \\
\hline Female, \% (n) & $30(49)$ & $32(14)$ & $35(22)$ & $23(13)$ \\
\hline High school graduate or equivalent & $63(103)$ & $52(23)$ & $70(44)$ & $63(36)$ \\
\hline Health insurance, \% (n) & $92(150)$ & $84(37)$ & $95(60)$ & $93(53)$ \\
\hline Charlson comorbidity score, greater or equal to $1, \%(n)$ & $88(54)$ & $25(57)$ & $34(54)$ & $29(51)$ \\
\hline Marijuana & $60(98)$ & $48(21)$ & $43(27)$ & $88(50)$ \\
\hline Opioids & $13(22)$ & $14(6)$ & $19(12)$ & $7(4)$ \\
\hline Cocaine & $24(40)$ & $36(16)$ & $33(21)$ & $5(3)$ \\
\hline Other drugs & $2(4)$ & $2(1)$ & $5(3)$ & $0(0)$ \\
\hline Unhealthy drinking, any, \% (n)ף & $60(99)$ & $71(31)$ & $59(37)$ & $54(31)$ \\
\hline
\end{tabular}

Table 2 Baseline characteristics of participants with diabetes, overall and stratified by number of days of drug use in the past 30 days* $^{*}$

\begin{tabular}{|c|c|c|c|c|}
\hline \multirow[b]{2}{*}{ Characteristic } & \multirow[b]{2}{*}{ All $(n=62)$} & \multicolumn{3}{|c|}{$\begin{array}{l}\text { Number of days of drug use in past } 30 \text { days } \\
\text { (tertiles)t }\end{array}$} \\
\hline & & Lowest $(n=20)$ & Middle $(n=21)$ & Highest $(\mathrm{n}=21)$ \\
\hline Age, mean (SD) & $48(10)$ & $49(9)$ & $50(10)$ & $45(12)$ \\
\hline Female, \% (n) & $34(21)$ & $30(6)$ & $29(6)$ & $43(9)$ \\
\hline Non-White, $\%(n) \ddagger$ & $89(55)$ & 95 (19) & 91 (19) & $81(17)$ \\
\hline High school graduate or equivalent & $73(45)$ & $70(14)$ & $76(16)$ & $71(15)$ \\
\hline Health insurance, $\%(n)$ & $90(56)$ & $85(17)$ & $95(20)$ & $91(19)$ \\
\hline Charlson comorbidity score, greater or equal to $1, \%(n)$ & $98(61)$ & $100(20)$ & $100(21)$ & $95(20)$ \\
\hline Depression, \% (n)§ & $31(19)$ & $25(5)$ & $29(6)$ & $38(8)$ \\
\hline \multicolumn{5}{|l|}{ Drug type, \% (n)ף } \\
\hline Marijuana & $63(39)$ & $45(9)$ & $57(12)$ & $86(18)$ \\
\hline Opioids & $15(9)$ & $15(3)$ & $14(3)$ & $14(3)$ \\
\hline Cocaine & $18(11)$ & $35(7)$ & $19(4)$ & $0(0)$ \\
\hline Other drugs & $5(3)$ & $5(1)$ & $10(2)$ & $0(0)$ \\
\hline Unhealthy drinking, any, $\%(n)^{\star *}$ & $60(37)$ & $65(13)$ & $62(13)$ & $52(11)$ \\
\hline ASSIST global drug score, mean (std)†† & $16(13)$ & $10(9)$ & $19(15)$ & $19(14)$ \\
\hline 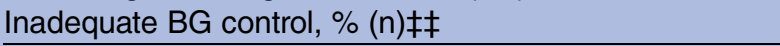 & $44(24)$ & $53(8)$ & $45(9)$ & $37(7)$ \\
\hline \multicolumn{5}{|c|}{ 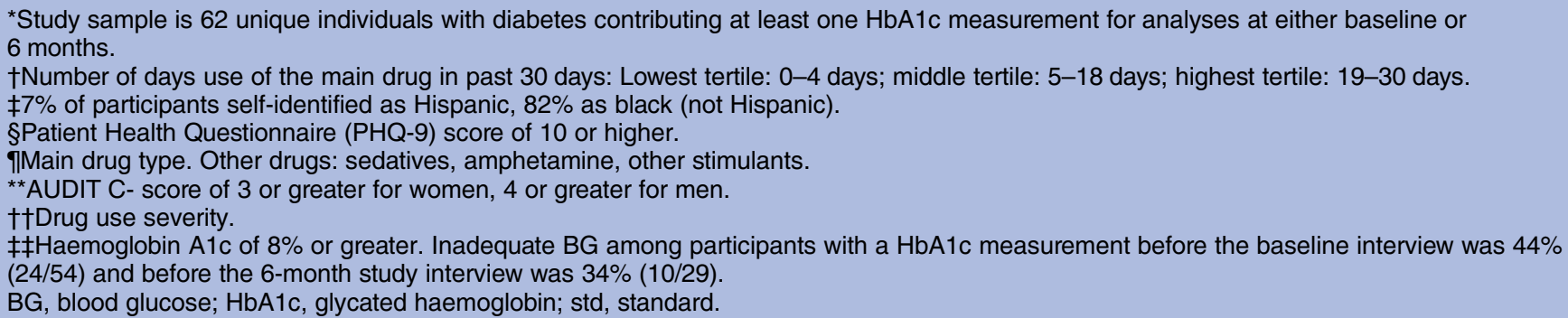 } \\
\hline
\end{tabular}

Number of days of drug use in past 30 days (tertiles) $^{\star}$

\section{Characteristic}

Female, \% (n)

Non-White, \% (n)

Health insurance, \% (n)

Depression, \% (n):

Opioids

Other drugs

Unhealthy drinking, any, \% (n)

Inadequate BP control, \% (n)†† 
the participants with hypertension, followed by cocaine $(24 \%)$ and opioids $(13 \%)$. Similar proportions of the type of main drug were found in the diabetes subsample. Overall, $40 \%(65 / 164)$ of participants with hypertension and BP data at baseline had inadequate BP control. Forty-four per cent $(24 / 54)$ of the sample with diabetes and a HbAlc measurement at baseline had inadequate BG control. In the hypertension and diabetes analytic samples, marijuana was the main drug for almost all participants in the highest tertile of days of drug use.

\section{Associations between drug use measures and BP and BG control}

Table 3 presents the results of regression analyses examining the association between each drug use measure and $\mathrm{BP}$ control. The number of days of drug use was not significantly associated with inadequate $\mathrm{BP}$ control (adjusted OR (AOR) $0.67 ; 95 \%$ CI 0.31 to 1.46 ) highest versus lowest tertile; AOR 0.72; 95\% CI 0.36 to 1.41 middle versus lowest tertile in the final adjusted model. Results were similar across unadjusted and adjusted models. No significant associations were detected for drug type or drug severity and BP control, although the parameter estimates for higher severity and cocaine/ opioids were in the hypothesised direction (ie, greater odds of having inadequate BP control with higher severity of use). There were no significant interactions between number of days of drug use and drug type, unhealthy alcohol use or depression. Adjustment for randomisation group and analysing systolic BP control as a continuous measure resulted in similar conclusions (data not shown).
With regard to the diabetes results, the number of days of drug use was not significantly associated with BG control (table 4). However, cocaine use was significantly associated with greater odds of having inadequate $\mathrm{BG}$ control compared to those with marijuana use (AOR 8.82, $95 \%$ CI 1.86 to 41.90 ) in analyses adjusted for age, gender and race. Worse severity of drug use was also associated with higher odds of having inadequate BG control in an unadjusted model (OR 3.05; 95\% CI 1.01 to 9.23 ); however, this association was attenuated and no longer statistically significant after controlling for age, gender and race/ethnicity. Adjusting for randomisation group and examining BG control (ie, HbAlc) as a continuous variable revealed similar results (data not shown).

\section{DISCUSSION}

Among adults in primary care reporting recent drug use, we detected few associations between the number of days of drug use, drug type or drug use severity with hypertension and diabetes quality criteria for BP and BG control. Cocaine use appeared to be associated with worse BG control. The relationship between days of drug use and BP control did not appear to be moderated by drug type, unhealthy alcohol use or depression.

This study builds on prior studies that have found those with behavioural health conditions such as depression and substance use disorders are less likely to receive recommended diabetes care such as annual interval monitoring of $\mathrm{HbAlc}$, lipids, microalbumin and routine eye examinations. ${ }^{9} 1018$ Interestingly, one study using a

Table 3 Multivariable GEE logistic regression analysis evaluating the association between measures of drug use and inadequate BP control among primary care patients with unhealthy drug use ( $n=229$ observations)*

\begin{tabular}{|c|c|c|c|c|c|c|}
\hline \multirow[b]{2}{*}{ Main independent variable } & \multicolumn{2}{|l|}{ Unadjusted models } & \multicolumn{2}{|l|}{ Adjusted models $†$} & \multicolumn{2}{|c|}{ Final adjusted model†’ł } \\
\hline & OR (95\% Cl) & p Value & OR (95\% Cl) & p Value & OR (95\% Cl) & p Value \\
\hline \multicolumn{7}{|c|}{ Number of days of drug use in past 30 days } \\
\hline Highest tertile (23-30 days) & $0.82(0.44$ to 1.52$)$ & 0.52 & $0.70(0.37$ to 1.32$)$ & 0.28 & $0.67(0.31$ to 1.46$)$ & 0.31 \\
\hline Middle tertile (3-22 days) & 0.88 (0.47 to 1.63$)$ & 0.68 & $0.82(0.43$ to 1.57$)$ & 0.55 & $0.72(0.36$ to 1.41$)$ & 0.34 \\
\hline Lowest tertile (0-2 days) & 1 & - & 1 & - & 1 & - \\
\hline \multicolumn{7}{|l|}{ Drug type } \\
\hline Cocaine & $1.44(0.75$ to 2.78$)$ & 0.28 & $1.44(0.75$ to 2.78$)$ & 0.28 & $1.12(0.52$ to 2.40$)$ & 0.78 \\
\hline Opioids & 1.61 (0.67 to 3.85$)$ & 0.28 & $1.76(0.71$ to 4.36$)$ & 0.22 & 1.77 (0.70 to 4.48$)$ & 0.23 \\
\hline Other & 0.81 (0.08 to 8.25$)$ & 0.86 & 1.00 (0.09 to 10.67$)$ & 0.998 & $0.84(0.07$ to 9.60$)$ & 0.89 \\
\hline Marijuana & 1 & - & 1 & - & 1 & - \\
\hline \multicolumn{7}{|l|}{ Drug use severity } \\
\hline Highest & $1.48(0.74$ to 2.93$)$ & 0.27 & 1.47 (0.69 to 3.12$)$ & 0.32 & $1.50(0.68$ to 3.30$)$ & 0.31 \\
\hline Middle & $0.99(0.51$ to 1.95$)$ & 0.99 & $0.97(0.49$ to 1.93$)$ & 0.93 & $1.14(0.53$ to 2.47$)$ & 0.74 \\
\hline Lowest & 1 & - & 1 & - & 1 & - \\
\hline
\end{tabular}

*Inadequate BP control defined as systolic BP of 140 or greater or diastolic BP of 90 or greater as specified by the National Committee for Quality Assurance (NCQA) and others. Three separate GEE (generalised estimating equations) logistic regression models evaluated the association between each drug use measure (days use of the main drug in past 30 days, drug type, and drug use severity) and inadequate BP control.

†Adjusted models included age, sex, race/ethnicity, health insurance, depression, and Charlson score.

fOne multivariable model with same covariates and all three drug use measures. Age was the only statistically significant covariate in the days of drug use model ( $\mathrm{AOR}=0.96 ; 95 \% \mathrm{Cl} 0.93$ to $0.997, \mathrm{p}=0.034$ ) and in the drug type model ( $\mathrm{AOR}=0.97 ; 95 \% \mathrm{Cl} 0.93$ to $0.99, \mathrm{p}=0.047$ ). There were no significant covariates in the drug use severity model or in the final adjusted model.

AOR, adjusted OR; BG, blood glucose; BP, blood pressure; GEE, generalised estimating equations. 
Table 4 Multivariable GEE logistic regression analysis evaluating the association between measures of drug use and inadequate $B G$ control $(n=83 \text { observations })^{*}$

\begin{tabular}{|c|c|c|c|c|}
\hline \multirow[b]{2}{*}{ Main independent variable } & \multicolumn{2}{|l|}{ Unadjusted models } & \multicolumn{2}{|l|}{ Adjusted models $†$} \\
\hline & OR $(95 \% \mathrm{Cl})$ & p Value & OR (95\% Cl) & p Value \\
\hline \multicolumn{5}{|c|}{ Number of days of drug use in past 30 days } \\
\hline Highest tertile (19-30 days) & $0.43(0.13$ to 1.41$)$ & 0.16 & $0.27(0.07$ to 1.10$)$ & 0.07 \\
\hline Middle tertile (5-18 days) & $0.93(0.33$ to 2.66$)$ & 0.90 & 1.01 (0.38 to 2.69$)$ & 0.99 \\
\hline Lowest tertile ( $0-4$ days) & 1 & - & 1 & - \\
\hline \multicolumn{5}{|l|}{ Drug type } \\
\hline Cocaine & $4.44(1.01$ to 19.54$)$ & 0.048 & $8.82(1.86$ to 41.90$)$ & 0.006 \\
\hline Opioids & 0.56 (0.13 to 2.32$)$ & 0.42 & $0.53(0.12$ to 2.33$)$ & 0.40 \\
\hline Other & $1.67(0.14$ to 20.08$)$ & 0.69 & $4.71(0.26$ to 86.80$)$ & 0.30 \\
\hline Marijuana & 1 & - & 1 & - \\
\hline \multicolumn{5}{|l|}{ Drug use severity $\ddagger$} \\
\hline Highest & 3.05 (1.01 to 9.23$)$ & 0.048 & 2.57 (0.79 to 8.33$)$ & 0.12 \\
\hline Middle & 2.05 (0.67 to 6.24$)$ & 0.21 & $1.68(0.48$ to 5.85$)$ & 0.42 \\
\hline Lowest & 1 & - & 1 & - \\
\hline \multicolumn{5}{|c|}{$\begin{array}{l}\text { *Inadequate BG control defined as glycated haemoglobin of } 8 \% \text { or greater as specified by the National Committee for Quality Assurance } \\
\text { (NCQA). Three separate GEE (generalised estimating equations) logistic regression models were used to evaluate the association between } \\
\text { each drug use measure (days use of the main drug in the past } 30 \text { days, drug type and drug use severity) and inadequate BG control. All } \\
\text { available data at baseline and } 6 \text {-month interview was analysed as repeated measures. } \\
\text { †Adjusted models included age, gender, race/ethnicity. Age was the only statistically significant covariate in the days of drug use model } \\
\text { (AOR=0.94 } 95 \% \mathrm{Cl} 0.89 \text { to } 0.99, \mathrm{p}=0.02 \text { ) and in the drug type model (AOR }=0.9395 \% \mathrm{Cl} 0.88 \text { to } 0.99, \mathrm{p}=0.02 \text { ). There were no significant } \\
\text { covariates in the analysis of drug use severity and inadequate BG control. } \\
\text { fAlcohol, Smoking, and Substance Involvement Screening Test (ASSIST) global drug score. } \\
\text { AOR, adjusted OR; BG, blood glucose; GEE, generalised estimating equations. }\end{array}$} \\
\hline
\end{tabular}

Medicaid data set found that substance use disorders were associated with lower receipt of process of care measures but not glycaemic control. ${ }^{18}$ One of the few studies examining primary care service delivery and the full spectrum of drug use found that those with unhealthy alcohol or drug use were less likely to receive breast cancer screening and flu vaccination but no difference was found for receipt of colon or cervical cancer screening. ${ }^{2}$ This study's finding of an association between cocaine use and glycaemic control is consistent with prior studies that found an association between cocaine use, missed insulin doses, excessive catecholamine production and poor glycaemic control. ${ }^{25} 26$

It is possible that despite the smaller sample size we detected an effect of drug use on BG control rather than BP control because drug use several weeks before the clinic visit may not be reflected by BP measured in the clinic but may affect a HgbAlc, a summary measure for the past few months. Time since last use may be another drug use measure to consider for BP control.

In models adjusted for frequency of use and other potential confounders, we did not detect a significant effect of marijuana use on hypertension control. Contrary to our hypothesis, greater frequency of drug use was not associated with greater likelihood of inadequate BG control. Instead greater drug use frequency appeared to be protective for BG control, although the result did not achieve statistical significance $(\mathrm{p}=0.07)$. It is important to note when interpreting this study's findings that the entire study sample was comprised of individuals with recent drug use. Comparisons made in this study were with other drug users, not individuals without any drug use, though the sample did include a wide range of drug use. It is possible that the effect of specific drugs may emerge more clearly with a comparison group using no drugs. Nonetheless, one would expect that if there were an association between drug use and chronic disease control measures, there would be a consistent dose-response type of association, with greater frequency and severity associated with worse outcomes.

Another consideration is the level of care provided by the primary care practice in this study. It is possible that this primary care practice had a greater capacity to deliver effective care for patients with more severe drug use and if so, then the results may not extend to other primary care practices. This possibility is given credence by the fact that the proportion of patients recruited from this safety-net primary care practice with good BP control is comparable to the 2012 national control rate (64\%) for members with known hypertension, insured by commercial health plans that report HEDIS measures $^{27}$ and better than the prevalence of inadequate BP control $(57 \%)$ in an older report about a VA population. ${ }^{28}$

This study has several limitations. First, the study was limited in power to detect differences due to the small sample size especially for the diabetes analyses. However, we note that the OR estimates were not uniformly in the direction of the hypothesised results. In fact, the point estimates for days of drug use and BP and BG control do not provide any signal that suggests higher frequency is associated with chronic disease measures. The limited sample size, particularly for the diabetes analysis, restricted our ability to adjust for all potential 
confounders. Thus, these study's findings should be viewed as hypothesis generating and may inform the design of larger studies.

Second, the study samples were comprised of patients presenting for a primary care visit with available data on $\mathrm{BP}$, or in the case of the diabetes, HbA1c. This may have biased the study sample towards participants who were more adherent to medical care. Even so, a substantial proportion of the sample did not have adequate $\mathrm{BP}$ or BG control: these are patients who might benefit from a primary care-delivered intervention to improve diabetes outcomes. Third, BP data were obtained during a medical visit in which methods for obtaining BP are not 'standardised' as might be the case in a research setting. Fourth, marijuana was the predominant drug of choice for a large proportion of the sample so examination of these issues in other samples with more stimulant or opioid use would be of interest. However, drug type was not a significant predictor of BP control and there were no significant interactions between days of drug use and drug type. Further, marijuana is likely the predominant drug used by other primary care populations. Additionally, drug use severity did not emerge as a significant predictor of outcomes, and there was a low correlation between drug type and frequency of use (0.26). Finally, this study's findings may not generalise to patients not enrolled or unwilling to enrol in a clinical trial, although recruitment occurred by universal screening with few exclusion criteria to comprise a 'real life' study sample of patients in primary care.

This study has several strengths. We examined actual disease control, a clinically significant but a more difficult performance measure to achieve than receipt of recommended care such as routine eye or podiatry care. $^{9} 1018$ This study had data on the full spectrum of unhealthy drug use, information not obtainable with administrative data. Instead, drug use was identified and assessed with validated instruments in patients with hypertension or diabetes, groups not adequately studied.

\section{CONCLUSIONS}

Disparities in chronic medical care have been documented for those with unhealthy alcohol use, yet data on unhealthy drug use is limited. These results provide information about primary care patients with chronic medical conditions and unhealthy drug use, a group that has not been well-studied despite the growing recognition of the need for strategies to address chronic conditions in patients with multimorbidity. ${ }^{8}{ }^{29}$ Our study suggests that ascertaining the frequency of drug use may not facilitate improvement in BP or BG control although it raises the possibility that particular illicit drugs, specifically cocaine, may portend worse diabetes control. It also remains possible that the effects of drug use on chronic disease control are limited to those with more severe unhealthy use. Further examination of the impact of drug use on important disease outcomes in larger cohorts is needed given the prevalence of drug use and the growing number of adults with chronic medical conditions.

Contributors TWK conceived the paper, the scope of which was refined by all coauthors, drafted and revised the manuscript. JHS, DMC, JB, JG, NW and RS conducted the parent study. DMC led the development of the analytic strategy and NW performed the analysis. All authors contributed to the design of the study, methods, interpretation of results; made substantive suggestions for revisions of the manuscript; and approved the final version.

Funding The study was funded by an award from the National Institute on Drug Abuse (R01 DA025068), a portion of which was funded by the Center for Substance Abuse Treatment, Substance Abuse and Mental Health Services Administration (SAMHSA).

Disclaimer Neither the National Institutes of Health $(\mathrm{NIH})$ nor SAMHSA contributed to the design and conduct of the study; collection, management, analysis, and interpretation of the data; and preparation, review, or approval of the manuscript; or the decision to submit the manuscript for publication.

Competing interests None declared.

Ethics approval Boston University School of Medicine.

Provenance and peer review Not commissioned; externally peer reviewed.

Data sharing statement No additional data are available.

Open Access This is an Open Access article distributed in accordance with the Creative Commons Attribution Non Commercial (CC BY-NC 4.0) license, which permits others to distribute, remix, adapt, build upon this work noncommercially, and license their derivative works on different terms, provided the original work is properly cited and the use is non-commercial. See: http:// creativecommons.org/licenses/by-nc/4.0/

\section{REFERENCES}

1. Substance Abuse and Mental Health Services Administration. Results from the 2013 National Survey on Drug Use and Health: Summary of National Findings, NSDUH Series H-48, HHS Publication No. (SMA) 14-4863. Rockville, MD: Office of Applied Studies, 2014.

2. Lasser KE, Kim TW, Alford DP, et al. Is unhealthy substance use associated with failure to receive cancer screening and flu vaccination? A retrospective cross-sectional study. BMJ Open 2011;1:e000046. http://bmjopen.bmj.com/content/1/1/e000046.long (accessed 1 Jun 2014).

3. Fuster D, Cheng DM, Allensworth-Davies D, et al. No detectable association between frequency of marijuana use and health or healthcare utilization among primary care patients who screen positive for drug use. J Gen Intern Med 2014;29:133-9.

4. Rosland AM, Nelson $\mathrm{K}$, Sun $\mathrm{H}$, et al. The patient-centered medical home in the Veterans Health Administration. Am J Manag Care 2013;19:e263-72.

5. Jaffe MG, Lee GA, Young JD, et al. Improved blood pressure control associated with a large-scale hypertension program. JAMA 2013;310:699-705.

6. National Committee for Quality Assurance (US). HEDIS, Washington D.C, Technical specifications. Improving quality and patient experience. The state of health care quality. 2013. http://www.ncqa. org/Portals/0/Newsroom/SOHC/2013/SOHC-web_version_report.pdf. (accessed 30 Jun 2014).

7. Khatib R, Schwalm JD, Yusuf S, et al. Patient and healthcare provider barriers to hypertension awareness, treatment and follow-up: a systematic review and meta-analysis of qualitative and quantitative studies. PLOS ONE 2014;9:e84238.

8. Piette JD, Kerr EA. The impact of comorbid chronic conditions on diabetes care. Diabetes Care 2006;29:725-31.

9. Desai MM, Rosenheck RA, Druss BG, et al. Mental disorders and quality of diabetes care in the Veterans Health Administration. Am J Psychiatry 2002;159:1584-90.

10. Druss BG, Zhao L, Cummings JR, et al. Medical comorbidity and quality of care under Medicaid: a 50 state analysis. Med Care 2012;50:428-33.

11. Ahmed AT, Karter AJ, Warton EM, et al. The relationship between alcohol consumption and glycemic control among patients with 
diabetes: the Kaiser Permanente Northern California Diabetes Registry. J Gen Intern Med 2008;23:275-82.

12. Thomas RM, Francis Gerstel PA, Williams EC, et al. Association between alcohol screening scores and diabetic self-behaviors. Fam Med 2012;44:555-63.

13. Merrill JO, Rhodes LA, Deyo RA, et al. Mutual mistrust in the medical care of drug users: the keys to the "narc" cabinet. J Gen Intern Med 2002;17:327-33.

14. Amaral-Sabadini MB, Saitz R, Souza-Formigoni ML. Do attitudes about unhealthy alcohol and other drug use (AOD) impact professionals' readiness to implement AOD-related preventive care? Drug Alcohol Rev 2010;29:655-61.

15. Wang EA, McGinnis KA, Goulet J, et al., Veterans Aging Cohort Study Project Team. Food insecurity and health: data from the Veterans Aging Cohort Study. Public Health Rep 2015;130: 261-8.

16. Meshesha LZ, Tsui J, Liebschutz JM, et al. Days of heroin use predict poor self-reported health in hospitalized heroin users. Addict Behav 2013;38:2884-7.

17. Rose AJ, Hermos JA, Frayne SM, et al. Does opioid therapy affect quality of care for diabetes mellitus? Am J Manag Care 2009;15:217-24.

18. Clark RE, Weir SW, Ouellette RA, et al. Beyond health plans. Behavioral health disorders and quality of diabetes and asthma care for Medicaid beneficiaries. Med Care 2009;47:545-52.

19. Saitz R, Palfai TP, Cheng DM, et al. Screening and brief intervention for drug use in primary care: the ASPIRE randomized clinical trial. JAMA 2014;312:502-13.
20. Humeniuk R, Ali R, Babor TF, et al. Validation of the alcohol, smoking and substance involvement screening test (ASSIST). Addiction 2008;103:1039-47.

21. Westerberg VS, Togigan JS, Miller WR. Reliability of form 90D: an instrument for quantifying drug use. Subst Abus 1998;19:179-89.

22. Chaudhry S, Jin L, Meltzer D. Use of a self-report-generated Charlson Comorbidity Index for predicting mortality. Med Care 2005;43:607-15.

23. Kroenke K, Spitzer RL, Williams JB. The PHQ-9: validity of a brief depression severity measure. J Gen Intern Med 2001;16:606-13.

24. Bush K, Kivlahan DR, McDonell MB, et al. The AUDIT alcohol consumption questions (AUDIT-C): an effective brief screening test for problem drinking. Ambulatory Care Quality Improvement Project (ACQUIP). Alcohol Use Disorders Identification Test. Arch Intern Med 1998;158:1789-95.

25. Nyenwe EA, Loganathan RS, Blum S, et al. Active use of cocaine: an independent risk factor for recurrent diabetic ketoacidosis in a city hospital. Endocr Pract 2007:13:22-9.

26. Warner EA, Greene GS, Buchsbaum MS, et al. Diabetic ketoacidosis associated with cocaine use. Arch Intern Med 1998;158:1799-802.

27. Patel MM, Datu B, Roman D, et al. Centers for Disease Control and Prevention (CDC). Progress of health plans toward meeting the million hearts clinical target for high blood pressure control-United States 2010-2012. MMWR Morb Mortal Wkly Rep 2014;63:127-30.

28. Borzecki AM, Wong AT, Hickey EC, et al. Hypertension control: how are we doing? Arch Intern Med 2003;163:2705-11.

29. Weiss CO, Varadhan R, Puhan MA, et al. Multimorbidity and evidence generation. J Gen Intern Med 2014;29:653-60. 